\title{
Is It a New Approach for Treating Senile Hypertension with Kidney-Tonifying Chinese Herbal Formula? A Systematic Review of Randomized Controlled Trials
}

\author{
Jie Wang, Xingjiang Xiong, and Xiaochen Yang \\ Department of Cardiology, Guang'anmen Hospital, China Academy of Chinese Medical Sciences, Beijing 100053, China \\ Correspondence should be addressed to Xiaochen Yang; avill1988@126.com
}

Received 4 December 2013; Revised 31 December 2013; Accepted 3 January 2014; Published 17 February 2014

Academic Editor: Bo Feng

Copyright (C) 2014 Jie Wang et al. This is an open access article distributed under the Creative Commons Attribution License, which permits unrestricted use, distribution, and reproduction in any medium, provided the original work is properly cited.

\begin{abstract}
Objective. To assess the effect of kidney-tonifying (KT) Chinese herbal formula for senile hypertension (SH). Methods. A total of five databases (CENTRAL, Pubmed, CBM, CNKI, and VIP) were searched for published and unpublished randomized controlled trials (RCTs) up to November 30th, 2013. We included RCTs that confoundedly addressed the effect of KT formula in the treatment of SH. Results. Six studies involving 527 people were included. There was no evidence that KT formula alone had superior effects to antihypertensive drugs in systolic blood pressure (MD: $-3.70(-11.47,4.07) ; P=0.35)$ and diastolic blood pressure (MD: -3.00 $(-7.26,-1.26) ; P=0.17)$. However, there was evidence that KT formula combined with antihypertensive drugs was a better treatment option than antihypertensive drugs alone in systolic blood pressure (WMD: $-8.69(-12.35,-5.02)$; $P<0.00001)$ but no significant difference in diastolic blood pressure (WMD: $-1.32(-7.93,-5.30) ; P=0.7)$. In addition, after several weeks of treatment, the level of blood lipid, endothelin, blood urea nitrogen, and creatinine decreased significantly $(P<0.05)$. Conclusion. Compared with antihypertensive drugs alone, KT formula combined with antihypertensive drugs may provide more benefits for patients with SH. The large-scale high-quality trials are warranted in future.
\end{abstract}

\section{Introduction}

Senile hypertension ( $\mathrm{SH})$, a common cardiovascular disease among senile people, refers to the condition in patients over 60 years old who have a diastolic blood pressure (DBP) $\geq 12 \mathrm{kPa}(90 \mathrm{mmHg})$ and a systolic blood pressure (SBP) $\geq 18.6 \mathrm{kPa}(140 \mathrm{mmHg})$ or only have isolated systolic hypertension. As the average age increases in China, SH incidence is rising yearly and up to $75 \%$ among people over 60 [1]. Among the old people, high blood pressure (BP) has been proved to directly increase cardiovascular risk about three or four times [2]. Increased SBP has been recommended to be the major criterion for diagnosis, staging, and therapeutic management of $\mathrm{SH}[3,4]$. Several lines of strong evidence support the initiative to emphasize SBP. From the perspectives of pathology, the prevalence of cardiac and vascular disease is associated with aging, increased stiffness of large arteries, increased SBP, and increased pulse pressure frequently [5]. From the perspectives of epidemiology, isolated systolic hypertension is the most common form of hypertension and is present in approximately two-thirds of hypertensive individuals $>60$ years of age [6]. From the perspectives of diagnosis, classification and staging of hypertension are more precise when systolic rather than diastolic BP is used as the principal criterion [7]. Preventing the complications of hypertension, including stroke, coronary artery disease, myocardial infarction, heart failure, and kidney failure, may potentially impact not only an individual's functional status but also their ability to live independently in the community [8]. Despite the increased absolute risk for cardiovascular events associated with hypertension in the elderly, significant numbers of individuals remain untreated or inadequately treated [9]. Clinical benefits of treatment of isolated systolic hypertension include reductions in stroke, myocardial infarction, heart failure, kidney failure, and overall cardiovascular disease morbidity and mortality. For treatment, because the course of illness is long and accompanied with various degrees of visceral lesions of the heart, brain, and kidney, it has been proved by clinical experience that the blood pressure cannot be made to decrease too fast nor 
decrease too slow so as to prevent the severe complications which may happen after a long-term high blood pressure [10].

To treat senile hypertension, most patients need combination therapy, which means to take at least two or three drugs for achieving target BP every day and night. In addition to small dosage of such antihypertensive drugs as captopril (12.5 mg), nifedipine (10 mg), and atenolol (12.5 mg), Chinese herbal medicine (CHM) can be prescribed based on traditional Chinese medicine (TCM) differentiation of the syndromes for lowering BP, improving the blood supply of the target organs, and relieving the clinical symptoms. With the increasing prevalence of hypertension, there is a growing tendency for patients, especially for senile patients, to turn to CHM $[11,12]$. Several TCM clinical and experimental studies, including a substantial number of randomized controlled trials (RCTs), have shown that CHM is effective and safe for the treatment of hypertension $[13,14]$. One of the most important reasons for CHM to treat senile hypertension and reduce its complications is that TCM characterizes treatment by syndrome differentiation [15]. According to theory of The Yellow Emperor's Inner Classic (Huáng Dì Nèi Jīng), the kidneydeficiency (includes kidney-qi deficiency, kidney-yin deficiency, and kidney-yang deficiency) begins to occur on the elderly and leads to some symptoms such as tinnitus, lumbago, and dizziness. The common ingredients of the prescription for kidney-deficiency include Lu Rong (Cornu Cervi Pantotrichum), Yin Yang Huo (Herba Epimedii), and Tu Si Zi (Semen Cuscutae) for tonifying kidney-yang.

Although treating senile hypertension with kidneytonifying (KT) Chinese herbal formula had experienced hundreds of years and seems to have good effects in clinical practice, the evidence examining the efficacy of KT formula for senile hypertension has never been systematically summarized. Thus, we performed this systematic review to critically assess the effect and safety of KT formula for the treatment of senile hypertension.

\section{Methods}

2.1. Eligibility Criteria. We included randomized controlled trials that compared KT formula with placebo or antihypertensive drugs in old patients (age $>60$ ) with essential hypertension (DBP $\geq 90 \mathrm{mmHg}$ and $\mathrm{SBP} \geq 140 \mathrm{mmHg}$ ) or these only have isolated systolic hypertension. The eligible comparisons include

(i) KT formula versus any current antihypertensive medications;

(ii) KT formula plus any current antihypertensive medications for senile hypertension versus antihypertensive medications alone;

(iii) KT formula versus placebo.

Our prespecified primary outcome is all-cause mortality, and secondary outcomes include blood pressure (BP), the level of blood lipids (BL), endothelin (ET), blood urea nitrogen (BUN), creatinine (Cr), and adverse events.
2.2. Search Strategy. Two authors (X. Xiong and X. Yang) searched online databases including the Cochrane Central Register of Controlled Trials (CENTRAL, 2013), Pubmed (1950-2013), Chinese Biomedical Literature Database (CBM, 2013), Chinese National Knowledge Infrastructure (CNKI, 2013), and Chinese Scientific Journal Database (VIP, 2013) up to November 30th, 2013. The English searching terms were used individually or combined including "senile hypertension", "Kidney-tonifying", "Chinese herbal formula", "randomized controlled trial", "controlled clinical trial", "randomly", "trial", "randomised", and "randomized". The Chinese searching terms were used individually or combined including those for the generic name of senile hypertension "Lao_nian_xing_gao_xue_ya", kidney-tonifying "Bu_shen", and randomized "sui_ji". No language restriction was applied. The detailed search strategy of each database is available as supporting information.

We also searched unpublished postgraduate theses in Chinese databases. The reference lists of all relevant papers found electronically were hand-searched. Meanwhile, experts in this field and relevant pharmaceutical companies were contacted for additional references or unpublished studies.

2.3. Data Collection. Two reviewers (X. Xiong and X. Yang) independently screened the titles, abstracts, and key words of each searched article for potentially eligible studies. The full-text articles were retrieved for further assessment if the information given suggests that the study (1) included old patients with essential hypertension, (2) compared KT formula with western medication in the presence or absence of cointerventions in both groups, (3) assessed one or more relevant clinical outcome measure such as morality and BP, and (4) used random allocation. The extracted information included study population; participant demographics and baseline characteristics; details of the intervention and control conditions; study methodology; outcome measures and main results. Any discrepancies were identified and resolved through discussion with a third author (J. Wang) if necessary.

2.4. Risk of Bias (Methodological Quality) Assessment. Two reviewers (X. Xiong and X. Yang) independently assessed the risk of bias for each trial according to the Cochrane Handbook for Systematic Reviews of Interventions version 5.1.0 [22]. The following items were assessed: random sequence generation (selection bias), allocation concealment (selection bias), blinding (performance bias and detection bias), incomplete outcome data (attrition bias), and selective outcome reporting (reporting bias). Based on Cochrane handbook, the risk of bias was categorized as low/unclear/high risk of bias. Trials which met all criteria were judged as having a low risk of bias, trials which met none of the criteria were judged as having a high risk of bias, and trials with insufficient information to judge were classified as unclear risk of bias. Disagreements were resolved by a third reviewer (J. Wang) when necessary.

2.5. Data Analysis. We performed meta-analyses using RevMan 5.1 software provided by Cochrane Collaboration for 
TABLE 1: Characteristics and methodological quality of included studies.

\begin{tabular}{|c|c|c|c|c|c|c|}
\hline Study ID & Sample & $\begin{array}{l}\text { Diagnosis } \\
\text { standard }\end{array}$ & Intervention & Control & $\begin{array}{l}\text { Course } \\
\text { (week) }\end{array}$ & $\begin{array}{l}\text { Outcome } \\
\text { measure }\end{array}$ \\
\hline Lu et al., 2007 [16] & 90 & JNC-7 & $\begin{array}{l}\text { KT formula plus } \\
\text { indapamide }\end{array}$ & $\begin{array}{l}\text { Placebo plus } \\
\text { indapamide }\end{array}$ & 24 & $\begin{array}{l}\text { BP; BL; adverse } \\
\text { effects }\end{array}$ \\
\hline Hu et al., 2001 [17] & 84 & $\begin{array}{l}\text { CGPMHBP- } \\
1987\end{array}$ & KT formula & Captopril & 2 & $\mathrm{BP} ; \mathrm{BL}$ \\
\hline Huang et al., 2007 [18] & 60 & $\begin{array}{c}1999 \text { WHO-ISH } \\
\text { GMH }\end{array}$ & $\begin{array}{l}\text { KT formula plus } \\
\text { amlodipine basylate }\end{array}$ & $\begin{array}{l}\text { Amlodipine } \\
\text { basylate }\end{array}$ & 2 & $\mathrm{BP} ; \mathrm{ET}$ \\
\hline Kong, 2003 [19] & 60 & $\begin{array}{c}1999 \text { WHO-ISH } \\
\text { GMH }\end{array}$ & $\begin{array}{l}\text { KT formula plus } \\
\text { plendil }\end{array}$ & Plendil & 4 & $\mathrm{BP}$ \\
\hline Zhang and Li, 2006 [20] & 136 & $\begin{array}{c}1999 \text { WHO-ISH } \\
\text { GMH }\end{array}$ & KT pill plus cilazapril & Cilazapril & 8 & BP; ET; BUN; Cr \\
\hline Zhang et al., 2008 [21] & 97 & $\begin{array}{l}\text { CGPMHBP- } \\
2005\end{array}$ & KT pill plus captopril & Captopril & 8 & BP; BUN; Cr \\
\hline
\end{tabular}

Abbreviations: KT: kidney-tonifying; WHO-ISH GMH, WHO-ISH guidelines for the management of hypertension; CGPMHBP: China Guidelines on Prevention and Management of High Blood Pressure; JNC-7: Seventh Report of the Joint National Committee on the Prevention, Detection, Evaluation, and Treatment of High Blood Pressure; BP: blood pressure; BL: blood lipid; ET: endothelin; BUN: blood urea nitrogen; Cr: creatinine.

data analyses. Dichotomous data were expressed as risk ratio (RR) for binary outcomes and mean difference (MD) for continuous outcomes, with their $95 \%$ confidence intervals (CI), respectively. If required data were not reported, we requested data from corresponding author. We used fixed effects model unless there was evidence of heterogeneity. The statistical heterogeneity was examined with the $I^{2}$-test, where $I^{2}$ values of $50 \%$ or more were considered to be indicator of a substantial heterogeneity. In the absence of significant heterogeneity, we pooled data using a fixed-effect model $\left(I^{2}<50 \%\right)$; otherwise we used random effects model $\left(I^{2}>50 \%\right)$ [22].

\section{Result}

A total of six trials [16-21], involving 527 participants with senile hypertension, were included in meta-analysis (see Table 1 and Figure 1). All 6 trials were conducted in China. The treatment duration ranged from 2 to 24 weeks. One trial [17] compared KT formula versus captopril. The other 5 trials compared KT formula plus western medications versus western medications alone. There was one placebo controlled study [16]. Only one trial [16] mentioned outcome measure of mortality and adverse events. All trials reported decreasing of BP. The compositions of different KT prescription were presented in Table 2.

Methodologically, all studies were at high risk of bias (see Table 3). One trial [16] described the method of randomization in detail, and the method was also appropriate. All the other studies did not report information on the allocation concealment. None were double blinded. The follow-up was recorded in one study [16]. No studies conducted intentionto-treat analysis.

3.1. Primary Outcomes. No trial adopted mortality as primary outcome. There was no report of the incidence of any heart event (e.g., acute myocardial infraction, severity arrhythmia, heart failure, and revascularization).

\subsection{Secondary Outcomes}

3.2.1. KT Formula versus Western Medications. One trial [17] compared KT formula alone versus western medicine. There was no significant difference between the two groups in systolic blood pressure (MD: $-3.70(-11.47,4.07) ; P=0.35)$ and diastolic blood pressure (MD: $-3.00(-7.26,-1.26) ; P=0.17$ ) after 24 weeks of treatment (see Table 4 ).

3.2.2. KT Formula Plus Western Medications versus Western Medications. A total of 5 trials [16, 18-21] compared the combination of modified KT formula plus antihypertensive drugs with antihypertensive drugs. Three trials $[16,19,20]$ showed that there was a significant difference between treatment and control group in systolic blood pressure (MD: $-8.69(-12.35$, $-5.02) ; P<0.00001$ ) but no significant difference between treatment and control group in diastolic blood pressure (MD: $-1.32(-7.93,-5.30) ; P=0.7)$ (see Table 5).

3.3. Other Outcomes (BL, ET, BUN, and Cr). Two trials $[16,17]$ showed that after treatment, the level of blood lipid (BL) decreased significantly $(P<0.05)$ in KT formula group. Two trials [18-20] showed that after 2-8 weeks of treatment, the level of endothelin (ET) decreased significantly $(P<0.01)$ in KT formula group plus western medications group compared to western medications group. Furthermore, two trials [20, 21] showed that after 8 weeks of treatment, the level of blood urea nitrogen (BUN) and creatinine $(\mathrm{Cr})$ decreased significantly $(P<0.05)$ in KT formula group plus western medications group compared to western medications group.

3.4. Adverse Effect. Only one trial mentioned the adverse effect in two groups such as palpitation, dizziness, and sleep [16]. These adverse effects disappeared after continuing to take medicine. The other five trials [17-21] reported no side effect in the KT formula group compared to antihypertensive drugs. 


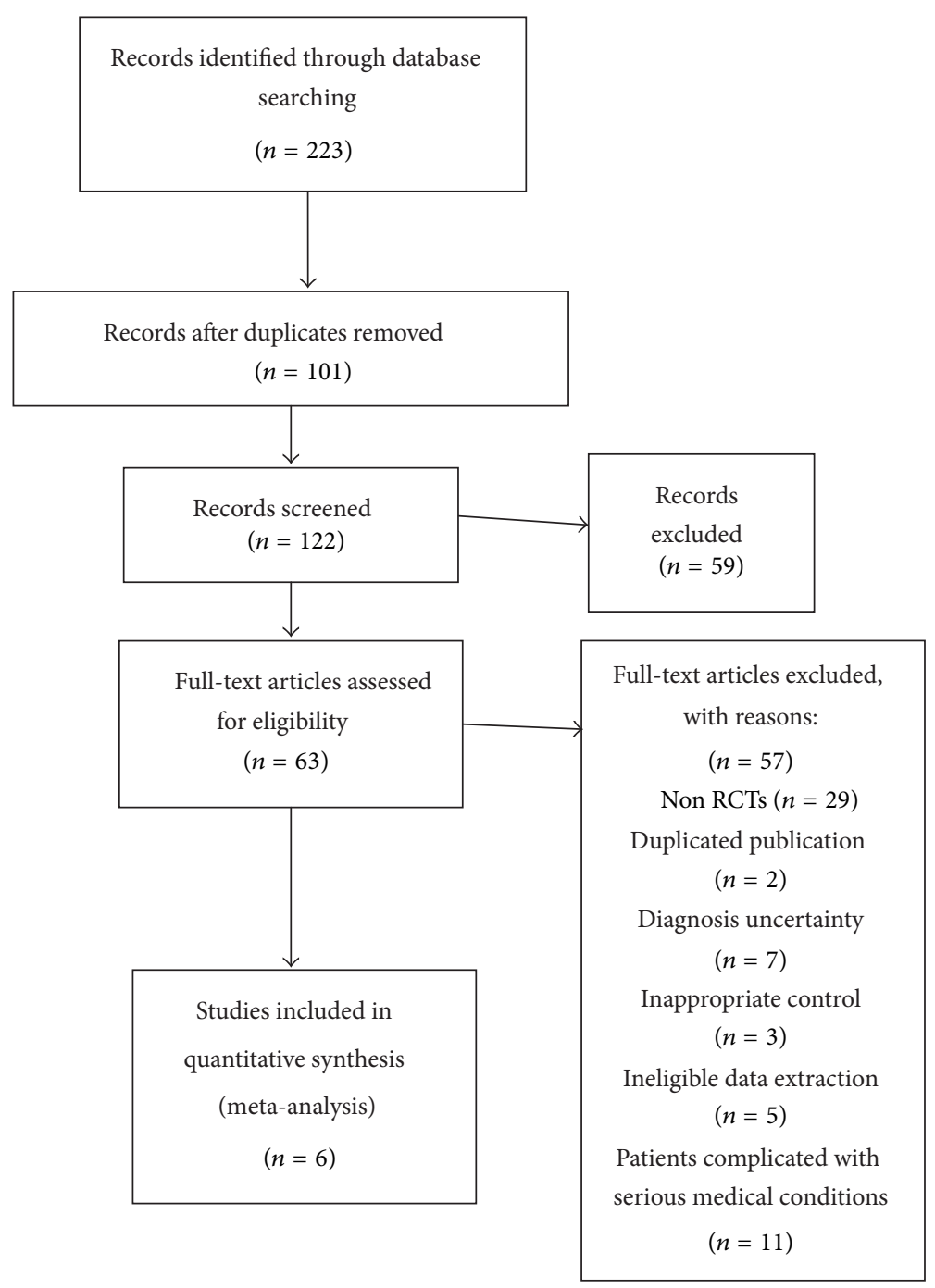

FIGURE 1: Flow Diagram of the literature searching and study selection.

TABLE 2: Assessment of risk of bias in included studies.

\begin{tabular}{|c|c|c|c|c|c|c|c|}
\hline Study & $\begin{array}{c}\text { Random } \\
\text { sequence } \\
\text { generation }\end{array}$ & $\begin{array}{l}\text { Allocation } \\
\text { concealment }\end{array}$ & Blinding & $\begin{array}{l}\text { Incomplete } \\
\text { outcome data }\end{array}$ & $\begin{array}{l}\text { Selective } \\
\text { reporting }\end{array}$ & $\begin{array}{c}\text { Free of other } \\
\text { biases }\end{array}$ & $\begin{array}{l}\text { Summary } \\
\text { assessments }\end{array}$ \\
\hline Lu et al., 2007 [16] & Low risk & Unclear & Unclear & Unclear & Unclear & Low risk & High risk \\
\hline Hu et al., 2001 [17] & Unclear & Unclear & Unclear & Unclear & Unclear & Unclear & High risk \\
\hline Huang et al., 2007 [18] & Unclear & Unclear & Unclear & Unclear & Unclear & Unclear & High risk \\
\hline Kong, 2003 [19] & Unclear & Unclear & Unclear & Unclear & Unclear & Unclear & High risk \\
\hline Zhang and Li, 2006 [20] & Unclear & Unclear & Unclear & Unclear & Unclear & Unclear & High risk \\
\hline Zhang et al., 2008 [21] & Unclear & Unclear & Unclear & Unclear & Unclear & Unclear & High risk \\
\hline
\end{tabular}

\section{Discussion}

In recent years, systematic reviews (SRs) and meta-analysis of CHM for treating hypertension have been conducted increasingly $[23,24]$. To the best of our knowledge, this is the first systematic review and meta-analysis of RCTs for KT formula in treating senile hypertension. Our results showed that KT formula may be good to lower BP, especially for SBP.
Based on the findings of meta-analyses, the preparation of KT formula used alone or combined with antihypertensive drugs may have some beneficial effects on the level of BL, ET, BUN, and $\mathrm{Cr}$ in old patients with essential hypertension. However, there were also some limitations of this review, which obstruct us to draw definite conclusion.

The most significant limitation is that all trials are at high risk of bias, which makes the findings less compelling. Except 
TABle 3: Composition of different KT prescriptions.

\begin{tabular}{|c|c|c|}
\hline Study ID & Preparation & Composition \\
\hline Lu et al., 2007 [16] & Decoction & $\begin{array}{l}\text { Cortex Eucommiae, Herba Taxilli, Fructus Ligustri Lucidi, Radix Astragali, Herba Epimedii, } \\
\text { Rhizoma Polygonati, Rhizoma Alismatis, Radix Cyathulae, Rhizoma Chuanxiong, Radix } \\
\text { Saposhnikoviae, Hirudo, Bombyx Batryticatus, and Rhizoma Gastrodiae (without details of } \\
\text { dose). For kidney-yang deficiency, Radix Aconiti Lateralis Praeparata and Herba Cistanches were } \\
\text { needed. For kidney-yin deficiency, Radix Rehmanniae Praeparata and Fructus Lycii were needed. }\end{array}$ \\
\hline Hu et al., 2001 [17] & Decoction & $\begin{array}{l}\text { Herba Taxilli } 15 \mathrm{~g} \text {, Herba Epimedii } 15 \mathrm{~g} \text {, Fructus Ligustri Lucidi } 15 \mathrm{~g} \text {, Radix et Rhizoma Salviae } \\
\text { Miltiorrhizae } 30 \mathrm{~g} \text {, Rhizoma Chuanxiong } 12 \mathrm{~g} \text {, Radix et Rhizoma Notoginseng } 5 \text { g, Radix } \\
\text { Puerariae Lobatae } 30 \mathrm{~g} \text {, Rhizoma Alismatis } 12 \mathrm{~g} \text {, and Poria } 12 \mathrm{~g} \text {. For kidney-yin deficiency, Radix } \\
\text { Rehmanniae Praeparata and Fructus Lycii were needed. For kidney-yang deficiency, Radix } \\
\text { Aconiti Lateralis Praeparata, Cortex Cinnamomi, and Herba Cistanches were needed. For yin } \\
\text { deficiency with yang hyperactivity, Radix Paeoniae Alba, and Rhizoma Gastrodiae were needed. }\end{array}$ \\
\hline Huang et al., 2007 [18] & Decoction & $\begin{array}{l}\text { Cortex Eucommiae } 15 \mathrm{~g} \text {, Herba Taxilli } 15 \text { g, Pheretima } 15 \text { g, Radix et Rhizoma Salviae } \\
\text { Miltiorrhizae } 15 \text { g, Herba Leonuri } 15 \text { g, Fructus Corni } 15 \text { g, and Cortex Moutan } 10 \text { g. }\end{array}$ \\
\hline Kong, 2003 [19] & Decoction & $\begin{array}{l}\text { Radix Rehmanniae } 15 \text { g, Radix Rehmanniae Praeparata } 15 \text { g, Polyporus, Poria } 15 \text { g, Rhizoma } \\
\text { Dioscoreae } 25 \text { g, Fructus Corni, Rhizoma Alismatis } 10 \text { g, Cortex Moutan } 10 \text { g, Radix Achyranthis } \\
\text { Bidentatae } 10 \text { g, Pheretima } 10 \text { g, Semen Plantaginis } 30 \text { g, and Radix et Rhizoma Salviae } \\
\text { Miltiorrhizae } 30 \text { g. For yin deficiency with yang hyperactivity, Rhizoma Gastrodiae } 15 \mathrm{~g} \text { and } \\
\text { Concha Margaritiferae Usta } 30 \text { g were needed. For deficiency of both yin and yang, Cortex } \\
\text { Cinnamomi } 3 \text { g and Radix Aconiti Lateralis Praeparata } 10 \text { g were needed. }\end{array}$ \\
\hline Zhang and Li, 2006 [20] & Pill & $\begin{array}{l}\text { Rhizoma Alismatis, Rhizoma Dioscoreae, Cortex Moutan, Fructus Corni, Poria, and Radix } \\
\text { Rehmanniae Recens (without details of dose). }\end{array}$ \\
\hline Zhang et al., 2008 [21] & Pill & $\begin{array}{l}\text { Rhizoma Alismatis, Rhizoma Dioscoreae, Cortex Moutan, Fructus Corni, Poria, and Radix } \\
\text { Rehmanniae Recens (without details of dose). }\end{array}$ \\
\hline
\end{tabular}

Abbreviations: KT: kidney-tonifying.

TABLE 4: Analyses of systolic blood pressure.

\begin{tabular}{|c|c|c|c|}
\hline Trials & & $\mathrm{MD}[95 \% \mathrm{CI}]$ & $P$ value \\
\hline \multicolumn{4}{|l|}{ KT versus antihypertensive drugs } \\
\hline KT formulae versus captopril & 1 & $-3.70[-11.47,4.07]$ & 0.35 \\
\hline Meta-analysis & 1 & $-3.70[-11.47,4.07]$ & 0.35 \\
\hline \multicolumn{4}{|c|}{ KT plus antihypertensive drugs versus antihypertensive drugs } \\
\hline KT formula plus felodipine versus felodipine & 1 & $-10.03[-15.53,-4.53]$ & 0.0004 \\
\hline KT formula plus indapamide versus indapamide & 1 & $-2.10[-9.85,5.65]$ & 0.60 \\
\hline KT pill plus cilazapril versus cilazapril & 1 & $-10.00[-11.79,-8.21]$ & $<0.00001$ \\
\hline Meta-analysis & 3 & $-8.69[-12.35,-5.02]$ & $<0.00001$ \\
\hline
\end{tabular}

Abbreviations: KT: kidney-tonifying.

TABle 5: Analyses of diastolic blood pressure.

\begin{tabular}{lcc}
\hline Trials & MD [95\% CI $]$ & $P$ value \\
\hline KT versus antihypertensive drugs & & $-3.00[-7.26,1.26]$ \\
KT formulae versus captopril & 1 & $-3.00[-7.26,1.26]$ \\
\hline Meta-analysis & 1 & 0.17 \\
\hline KT plus antihypertensive drugs versus antihypertensive drugs & & $-0.78[-2.61,4.17]$ \\
KT formula plus felodipine versus felodipine & 1 & $2.80[-1.30,6.90]$ \\
KT formula plus indapamide versus indapamide & 1 & $-7.00[-8.00,-5.20]$ \\
KT pill plus cilazapril versus cilazapril & 1 & $-1.32[-7.93,5.30]$ \\
\hline Meta-analysis & 3 & 0.18 \\
\hline
\end{tabular}

Abbreviations: KT: kidney-tonifying. 
for one trial [16], none of the other trials reported the method of randomization, they only mentioned that "patients were randomized into two groups", with no detailed information of randomization generation. Meanwhile, all trials claimed randomization, but they failed to provide enough information to judge whether the randomization procedures had been carried out properly. Only one placebo control was used [16], but none of the trials was of double-blind. In addition, no multicenter, large-scale RCTs were identified, and no dropouts and withdrawals were described. All the included trials used BP as outcome measure, but only half of the included trials evaluated the effectiveness with numerical values, which made the strengthening of evidence weak. It is recommended that future researchers should follow the basic guidelines for reporting clinical trials such as the Consolidated Standards of Reporting Trials (CONSORT) statement.

Several professional databases have been searched up to November 2013 including the Cochrane library, PubMed, CBM, CNKI, and VIP. After searching these databases, we found that all included trials were conducted and published in Chinese. The number of included trials is too small to conduct funnel plot analysis. So, the possibility of publication bias of $\mathrm{KT}$ formula for $\mathrm{SH}$ is unclear. In addition, there may be a limitation of missing some trials since we cannot search pertinent English databases like EMBASE.

Though none of the included trials reported severe adverse events possibly related to $\mathrm{KT}$ formula, we cannot draw firm conclusions about the safety of KT formula since seven trials did not report information on safety. The duration of treatment in most trials was 2 weeks or 8 weeks, so the potential toxin effect of KT formula for treatment of SH might only result from symptomatic changes (such as palpitation, dizziness, and sleep) and short treatment duration. The liver and kidney toxicity of Chinese medicines had drawn increasing attention to the concern of safety $[25,26]$. We recommend that further researchers should focus on the monitoring and reporting of adverse events and long-term safety by designing a longer duration of treatment and a long-term follow-up including health-related quality of life $[27,28]$.

\section{Conclusions}

Our review suggested that compared with antihypertensive medications alone, KT combined with western medications was of more benefits for patients with SH. However, considering the strength of the evidence, more rigorously designed, randomized double-blind placebo controlled trials are required for assessing the effects of KT formula before recommended routinely. Nevertheless, some aspects must be specially considered in future including methodological improvement, adverse effects, and reporting clinically outcomes from long-term follow-up. All of this needs more welldesigned, long-tern clinical trials.

\section{Conflict of Interests}

All authors declare that they have no conflict of interests.

\section{Author's Contribution}

J. Wang and X. Xiong contributed equally to this paper.

\section{Acknowledgments}

The current work was partially supported by the National Basic Research Program of China (973 Program, no. 2003CB517103) and the National Natural Science Foundation Project of China (no. 90209011). The funders had no role in study design, data collection and analysis, decision to publish, or preparation of the paper.

\section{References}

[1] H. Arima, Y. Murakami, T. Hing Lam et al., "Effects of prehypertension and hypertension subtype on cardiovascular disease in the Asia-pacific region," Hypertension, vol. 59, pp. 1118-1123, 2012.

[2] W. B. Kannel, T. Gordon, and M. J. Schwartz, "Systolic versus diastolic blood pressure and risk of coronary heart disease. The Framingham study," The American Journal of Cardiology, vol. 27, no. 4, pp. 335-346, 1971.

[3] H. P. Dustan, "50th anniversary historical article: hypertension," Journal of the American College of Cardiology, vol. 33, pp. 595$597,1999$.

[4] S. Madhavan, W. L. Ooi, H. Cohen, and M. H. Alderman, "Relation of pulse pressure and blood pressure reduction to the incidence of myocardial infarction," Hypertension, vol. 23, no. 3, pp. 395-401, 1994.

[5] M. O’Rourke, "Arterial stiffness, systolic blood pressure, and logical treatment of arterial hypertension," Hypertension, vol. 15, no. 4, pp. 339-347, 1990.

[6] V. L. Burt, J. A. Cutler, M. Higgins et al., "Trends in the prevalence, awareness, treatment, and control of hypertension in the adult US population: data from the health examination surveys, 1960 to 1991," Hypertension, vol. 26, pp. 60-69, 1995.

[7] National High Blood Pressure Education Program Working Group, "National High Blood Pressure Education Program Working Group report on hypertension in the elderly, Hypertension, vol. 23, pp. 275-285, 1994.

[8] A. Benetos, F. Thomas, K. Bean, S. Gautier, H. Smulyan, and L. Guize, "Prognostic value of systolic and diastolic blood pressure in treated hypertensive men: clarification," Archives of Internal Medicine, vol. 163, no. 1, article 121, 2003.

[9] J. A. Staessen, J. Gasowski, J. G. Wang et al., "Risks of untreated and treated isolated systolic hypertension in the elderly: metaanalysis of outcome trials," The Lancet, vol. 355, no. 9207, pp. 865-872, 2000.

[10] R. J. Glynn, G. J. L'Italien, H. D. Sesso, E. A. Jackson, and J. E. Buring, "Development of predictive models for long-term cardiovascular risk associated with systolic and diastolic blood pressure," Hypertension, vol. 39, no. 1, pp. 105-110, 2002.

[11] E. Ernst, "Prevalence of use of complementary/alternative medicine: a systematic review," Bulletin of the World Health Organization, vol. 78, no. 2, pp. 252-257, 2000.

[12] K. J. Chen, K. K. Hui, M. S. Lee, and H. Xu, “The potential benefit of complementary/alternative medicine in cardiovascular diseases," Evidence-Based Complementary and Alternative Medicine, vol. 2012, Article ID 125029, 10 pages, 2012. 
[13] J. Wang, X. J. Xiong, G. Y. Yang et al., "Chinese herbal medicine qi ju di huang wan for the treatment of essential hypertension: a systematic review of randomized controlled trials," EvidenceBased Complementary and Alternative Medicine, vol. 2013, Article ID 262685, 10 pages, 2013.

[14] X. J. Xiong, W. Liu, X. C. Yang et al., "Moxibustion for essential hypertension," Complementary Therapies in Medicine, 2013.

[15] J. Wang and X. Xiong, "Control strategy on hypertension in Chinese medicine," Evidence-Based Complementary and Alternative Medicine, vol. 2012, Article ID 284847, 6 pages, 2012.

[16] F. Lu, C. H. Yang, Z. Wang et al., "Clinical effect of BuShen HeLuo decoction on old patients with essential hypertension," He Nan Zhong Yi Za Zhi, vol. 27, no. 1, pp. 29-33, 2007.

[17] J. B. Hu, H. Y. Wang, and X. L. Huang, "The effect of BuShen HuoXue Decoction on elderly essential hypertension," Ji Lin Zhong Yi Yao Za Zhi, vol. 2, pp. 8-11, 2001.

[18] Y. P. Huang, J. F. Wu, and Z. Q. Chen, "Effects of BuShen HuoXue Decoction for old patients with essential hypertension," Journal of Fujian College of TCM, vol. 17, no. 2, pp. 3-5, 2007.

[19] L. J. Kong, "The effect of BuShen JiangYa Decoction on senile hypertension," Zhe Jiang Zhong Yi Za Zhi, vol. 20, no. 17, pp. 148150, 2003.

[20] T. D. Zhang and C. X. Li, "Clinical effect of Liu Wei Di Huang Wan for kidney protection on patients with senile hypertension," Zhong Yi Yao Xian Dai Hua Za Zhi, vol. 8, no. 2, pp. 102107, 2006.

[21] G. Q. Zhang, W. Y. Lai, K. Chen et al., "Clinical observation of Liu Wei Di Huang Wan combined with captopril for patients with senile hypertension," Journal of Hainan Medical College, vol. 14, no. 4, pp. 357-360, 2008.

[22] J. P. T. Higgins and S. Green, Corchrane Reviewers' Handbook 5.1.0, Review Manager (RevMan) [Computer program]. Version 5.1.0.

[23] J. Wang and X. J. Xiong, "Outcome measures of Chinese herbal medicine for hypertension: an overview of systematic reviews," Evidence-Based Complementary and Alternative Medicine, vol. 2012, Article ID 697237, 7 pages, 2012.

[24] J. Wang and X. J. Xiong, "Evidence-based Chinese Medicine for hypertension," Evidence-Based Complementary and Alternative Medicine, vol. 2013, Article ID 978398, 12 pages, 2013.

[25] J. S. Wang, R. van der Heijden, S. Spruit et al., "Quality and safety of Chinese herbal medicines guided by a systems biology perspective," Journal of Ethnopharmacology, vol. 126, no. 1, pp. 3141, 2009.

[26] K. Chan, "Some aspects of toxic contaminants in herbal medicines," Chemosphere, vol. 52, no. 9, pp. 1361-1371, 2003.

[27] J. Wang and X. J. Xiong, "Current situation and perspectives of clinical study in integrative medicine in China," Evidence-Based Complementary and Alternative Medicine, vol. 2012, Article ID 10.1155/2012/268542, 11 pages, 2012.

[28] X. J. Xiong, X. C. Yang, Y. M. Liu, Y. Zhang, P. Q. Wang, and J. Wang, "Chinese herbal formulas for treating hypertension in traditional Chinese medicine: perspective of modern science," Hypertension Research, vol. 36, no. 7, pp. 570-579, 2013. 


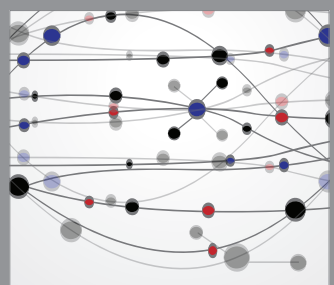

The Scientific World Journal
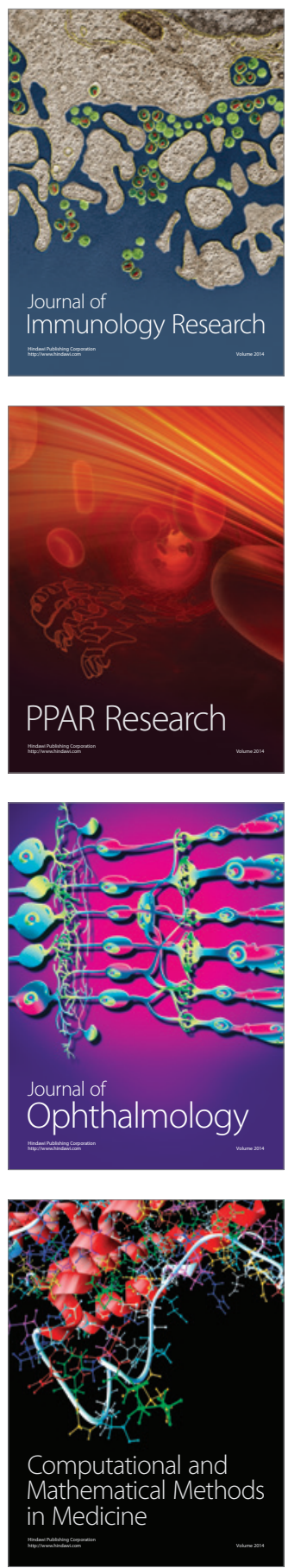

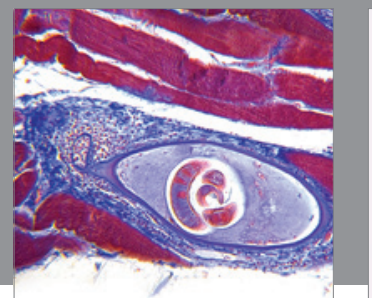

Gastroenterology

Research and Practice
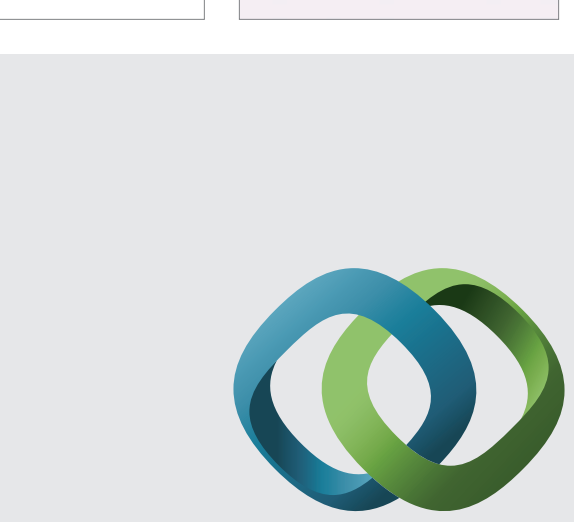

\section{Hindawi}

Submit your manuscripts at

http://www.hindawi.com
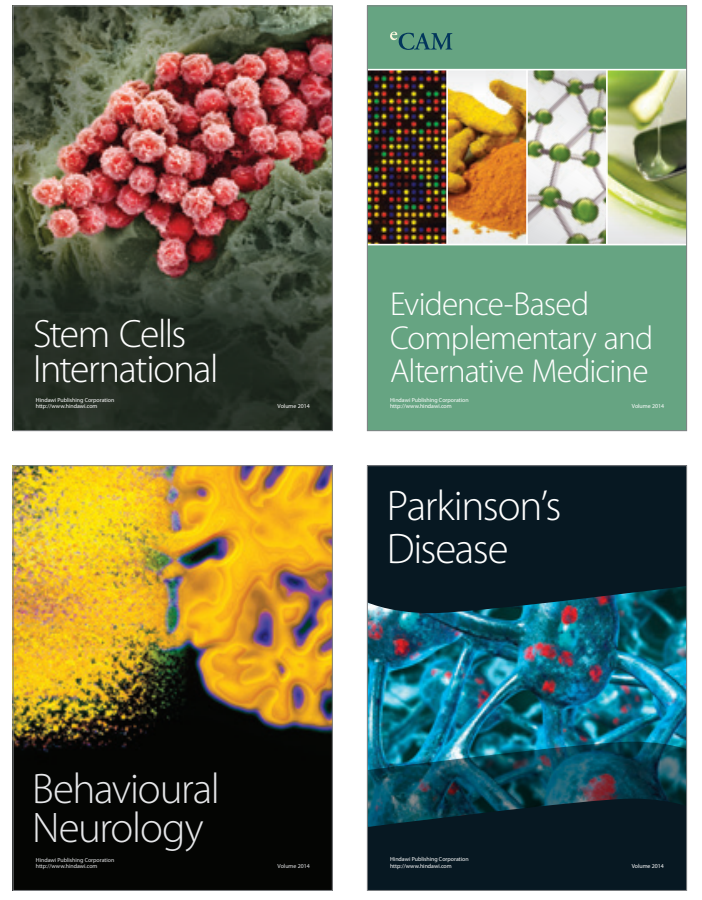
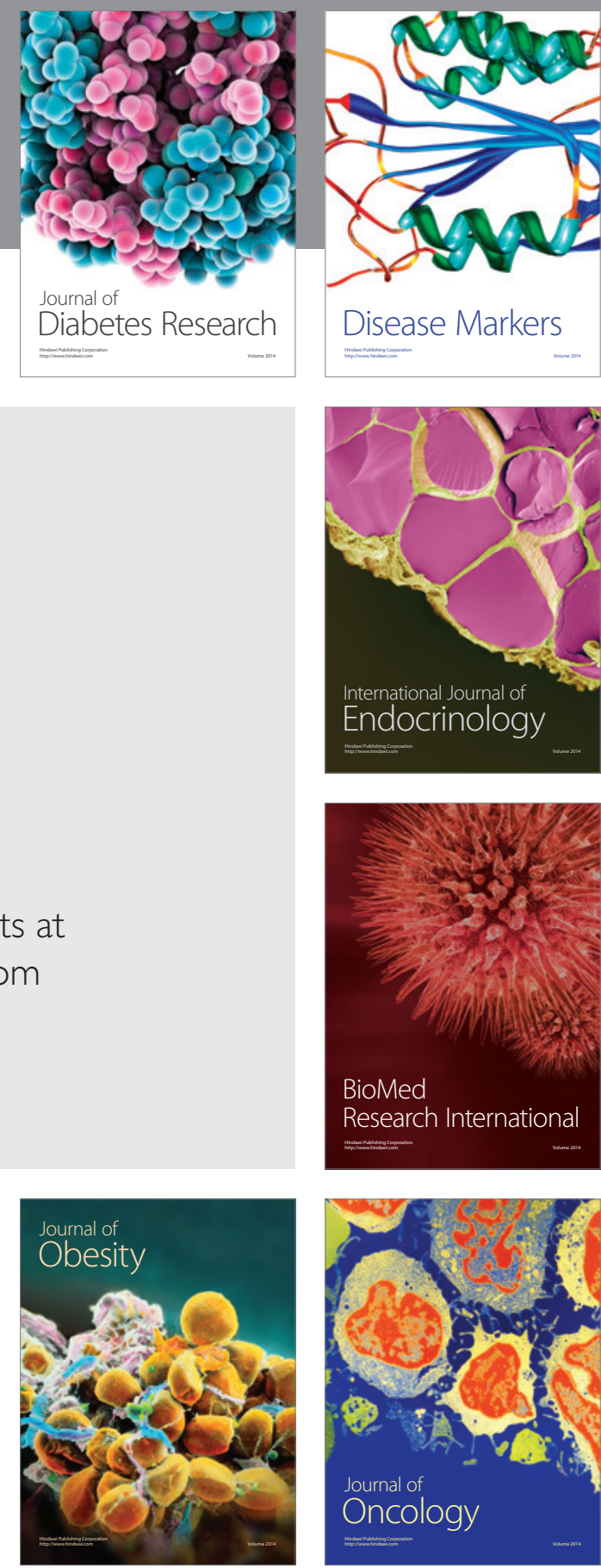

Disease Markers
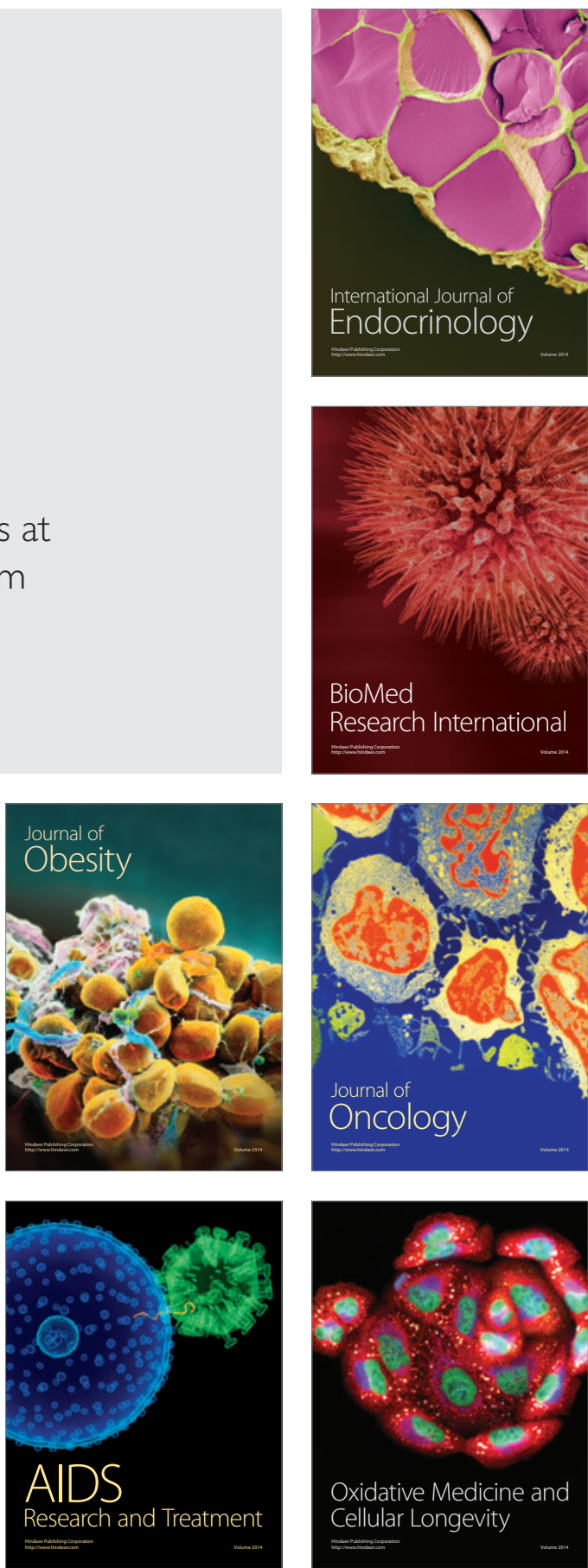